\title{
Carvão ativado obtido de resíduos agrícolas: adsorvente para remoção de corantes orgânicos dos efluentes têxteis
}

\author{
Cristiele Costa de Souza ${ }^{1}$ \\ Alan Rodrigues Teixeira Machado ${ }^{2}$
}

\begin{abstract}
RESUMO
A indústria têxtil é responsável por consumir grandes volumes de água e produtos químicos - por exemplo, os corantes. Isso gera efluentes com alta complexidade que podem contaminar os corpos hídricos se não forem tratados de forma adequada. Neste sentido, ganham destaque os métodos e materiais para o tratamento dos efluentes desse segmento. A adsorção com carvão ativado tem sido relatada com frequência para contornar esse problema. Contudo, o custo com esse insumo tem sido um entrave para muitas empresas. Assim, muitos grupos de pesquisas têm se dedicado à busca por novas fontes de matérias-primas para a produção do carvão ativado. Neste artigo será apresentado o resultado de um levantamento de fontes de resíduos agrícolas que foram submetidos à pirólise e caracterizados quanto ao potencial adsortivo de corantes. Para tanto, utilizou-se a base de dados Web of Science ${ }^{\mathrm{TM}}$ e delimitou-se como critério de inclusão apenas as referências dos últimos 5 anos associadas a resíduos agrícolas e corantes da indústria têxtil. Os resultados mostraram uma gama de resíduos agrícolas empregados para a produção de carvão ativado e com potencial para remoção de corantes têxteis.
\end{abstract}

Palavras-chave: Resíduos Agrícolas. Adsorção. Corantes. Carvão Ativado.

\footnotetext{
${ }^{1}$ Graduada em Engenheira Ambiental pelo Centro Universitário de Belo Horizonte - UniBH. Mestranda em Ciências Ambientais pela Universidade do Estado de Minas Gerais - UEMG, Unidade de Frutal, MG, Brasil. E-mail: tielle09@hotmail.com.

${ }^{2}$ Graduado em Química e Mestre em Agroquímica, ambos pela Universidade Federal de Lavras - UFLA. Doutor em Ciências (Química) pela Universidade Federal de Minas Gerais - UFMG. Possui capacitação para o Ensino de Empreendedorismo pela Babson College, EUA. É professor da Universidade do Estado de Minas Gerais - UEMG, Unidade João Monlevade, do Centro Universitário de Belo Horizonte - UniBH e orientador permanente do Programa de Pós-Graduação em Ciências Ambientais da UEMG, Unidade de Frutal, MG, Brasil. E-mail: alanvisconde@gmail.com.
} 


\title{
Agricultural wastes as a source for activated carbon production: adsorbent for organic dye removal
}

\begin{abstract}
The textile industry is responsible for consuming large volumes of water and chemicals -for example, dyes. These results in the generation of highly complex effluents that, if not correctly treated, can pollute and contaminate water bodies. In this sense, the methods and materials for the treatment of effluents in this segment stand out. Activated carbon adsorption has often been reported to circumvent this problem. But the cost of this input has been a drag on many companies. Thus, several researchers are dedicated to search for new sources of raw materials to produce activated carbon. In this paper, we present the results of a survey of agricultural waste sources that were pyrolyzed and characterized by their adsorptive potential. For this, we used the Web of Science ${ }^{T M}$ database, and only the references of the last five years, those associated with agricultural residues and dyes of the textile industry, were delimited as inclusion criteria. The results showed a range of agricultural residues used for the production of activated charcoal and with potential for textile dye removal.
\end{abstract}

Keywords: Agricultural Waste. Adsorption. Dyes. Activated Carbon.

Artigo recebido em: 01/05/2020

Aceito em: 01/06/2020 


\section{INTRODUÇÃO}

Os corpos hídricos sempre foram utilizados para o despejo sem tratamento de dejetos humanos e efluentes industriais, acarretando sua deterioração e má qualidade. O setor industrial é responsável por consumir grandes volumes de água e utilizar diversos compostos orgânicos e inorgânicos na fabricação de seus produtos (ADEKOGE; BELLO, 2015). Nesse contexto, destaca-se a indústria têxtil, a qual consome quase $200 \mathrm{~L}$ de água para fabricar 1 kg de tecido (YASEEN; SCHOLZ, 2019), incorporando também, à produção, dois terços de corantes produzidos no mundo (RANGABHASHIYAM; ANU; SELVARAJU, 2013). No seu processamento por via úmida, principalmente no tingimento, efluentes são descartados contendo elevada carga de insumos químicos que contribuem para alta taxa da demanda química de oxigênio (DQO) e da demanda bioquímica de oxigênio (DBO) (SHANKER; RANI; JASSAL, 2017; CROCE et al., 2017; YASEEN; SCHOLZ, 2019). Dentre os compostos existentes nos efluentes, enfatizam-se os corantes.

Os corantes têxteis são classificados de acordo com a fixação à fibra ou por sua fórmula estrutural química (SHANKER; RANI; JASSAL, 2017). Devido a sua composição química, podem ser considerados carcinogênicos, mutagênicos e tóxicos. A presença nos corpos d'água impacta o processo de fotossíntese, reduz a oxigenação e pode causar microtoxicidade aos organismos aquáticos (RANGABHASHIYAM; ANU; SELVARAJU, 2013).

Para contornar esses problemas, existem técnicas para o tratamento dos efluentes, tais como: coagulação, oxidação, troca iônica, osmose reversa, separação por membranas, filtração, degradação anaeróbica, adsorção, entre outras (BRILLAS; MARTÍNEZ-HUITLE, 2015). Em razão da composição das águas residuais, não existe uma técnica definida (YASEEN; SCHOLZ, 2019), porém o método de adsorção apresenta eficiência na remoção de uma variedade de poluentes, eliminando-os sem deixar traços na solução (RANGABHASHIYAM; ANU; SELVARAJU, 2013).

O método de adsorção baseia-se na utilização (normalmente) de um sólido (adsorvente) que na sua superfície concentra determinada substância, denominada de adsorvato, existente tanto em fluidos líquidos como gasosos (NASCIMENTO et al., 2014). Entre os adsorventes empregados, o Carvão Ativado (CA) apresenta melhor desempenho, em virtude da sua estrutura porosa e área superficial. Para obter essas propriedades, a produção do CA pode ocorrer por ativação física ou química (ADEKOGE; BELLO, 2015; 
GISI et al., 2016). Entretanto, ainda que apresente alta capacidade na remoção de diferentes compostos, o seu uso sofre restrição por causa do alto custo de produção.

Nesse seguimento, a busca de novos materiais adsorventes, bem como de novas fontes carbonáceas para produção do CA, com baixo custo, cresceu significativamente (Figura 1). Para exemplificar, pode-se citar as pesquisas que empregaram casca de pepino (SMITHA et al., 2017), casca de caju (SUBRAMANIAM; PONNUSAMY, 2015), Lantana camara (GAUTAM et al., 2015) e sementes de Gossypium hirsutu (SIVARAJASEKAR et al., 2017). Os estudos descreveram o potencial dessas matérias-primas para a produção de carvão ativado, exibindo valores elevados para área superficial específica, volume de poros e da capacidade adsortiva de diferentes compostos.

Figura 1: Número de publicações relatadas com o termo "activated carbon"

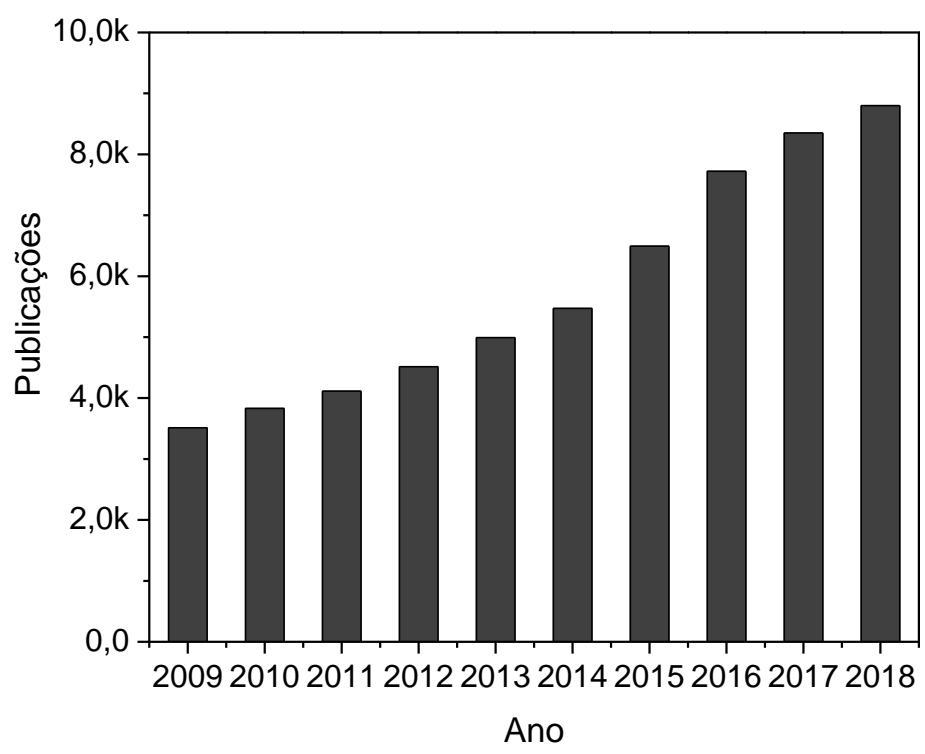

Fonte: Web of Science ${ }^{\mathrm{TM}}$ (2009-2018).

Diante do exposto, este trabalho tem por objetivo apresentar informações qualitativas e quantitativas sobre a capacidade adsortiva de carvões ativados produzidos a partir de resíduos agrícolas, contextualizando isso com a problemática dos efluentes aquosos que contém corantes orgânicos.

\section{METODOLOGIA}

A busca pelos artigos foi realizada na base de dados Web of Science ${ }^{\mathrm{TM}}$ (Coleção Principal da Thomson Reuters Scientific), uma vez que essa base possui ferramentas que 
permitem avaliar citações, referências, índice-h, bem como realizar análise bibliométrica de aproximadamente 12.000 periódicos (CAPES, 2019).

A procura do material bibliográfico consistiu em utilizar a aba pesquisa avançada, com a inserção no campo da expressão TS=(activated carbon* and adsorbents* and dyes*), estipulando o tempo para os últimos 5 anos. A pesquisa resultou em 639 referências. Posteriormente, refinou-se por artigos e números de citações, aparecendo o total de 577. Para escolha das referências, determinaram-se dois critérios: 1) matéria-prima transformada em carvão ativado; 2) adsorção de corantes da indústria têxtil. Após a definição, realizou-se avaliação dos resumos que consistiram em 89 artigos que atenderam aos limites estabelecidos, porém não se obteve acesso; 191 dos que estavam disponibilizados não atendiam às exigências; e 250 a que não se obteve acesso e que também não estavam dentro dos critérios. Assim, no primeiro momento selecionou-se 47 artigos, porém muitos relatavam apenas a secagem de resíduos agrícolas. Portanto, desse total, foram selecionados 21 artigos que realizavam a transformação de matérias-primas em carvão ativado.

\section{REVISÃO BIBLIOGRÁFICA}

\subsection{Indústria Têxtil}

O setor têxtil presente tanto em países desenvolvidos como em desenvolvimento tem por função suprir a demanda da população por produtos de vestuário e confecção. Estima-se que a produção alcance mais de 89 milhões de toneladas ao ano (YACOUT; HASSOUNA, 2016). Em toda sua cadeia produtiva são empregadas várias etapas, entre essas, a por via úmida, que utiliza grandes volumes de água e de insumos químicos, tais como: corantes orgânicos e sintéticos, surfactantes, agentes tensoativos, sais inorgânicos e diversos aditivos (YASEEN; SCHOLZ, 2019; SHANKER; RANI; JASSAL, 2017). Por consequência, os efluentes são caracterizados por serem coloridos com elevada temperatura, $\mathrm{pH}$, turbidez, alta carga da demanda química de oxigênio (DQO) e da demanda bioquímica de oxigênio (DBO) (CHICATTO et al., 2018; YASEEN; SCHOLZ, 2019).

As águas residuais têxteis, por conterem diferentes compostos, apresentam complexidade e diversidade química (DASGUPTA et al., 2015). Assim, o lançamento em corpos d'água com tratamento ineficiente/inexistente acarreta contaminação e poluição de rios, destruição da biota aquática, inibição da fotossíntese e redução de oxigênio 
(RANGABHASHIYAM; ANU; SELVARAJU, 2013; WANASSI et al., 2017). Na saúde humana, devido à presença de metais, os impactos são intensificados, tais como: dermatite alérgica, náuseas, diarreia, anemia, vômitos, aumento da frequência cardíaca, icterícia, cianose, fibrose pulmonar, doenças cardiovasculares, doenças renais e câncer (SHANKER; RANI; JASSAL, 2017; ZHOU; ZHANG; CHENG, 2015). Além desses impactos, existe também a geração de resíduos sólidos e emissões atmosféricas que é descrita em diversas pesquisas.

Dadi et al. (2017) analisaram o impacto tanto ambiental como social de quatro indústrias têxteis, localizadas nas cidades de Gelan e Duken na Etiópia. Os autores notaram que os efluentes têxteis com tratamento ineficiente/inexistente estavam sendo descartados em áreas abertas, próximas à população, e apresentavam níveis de DBO, DQO e de sólidos suspensos totais acima do limite permitido pela Environmental Protection Authority (EPA). Além disso, observaram a presença da Escherichia coli (bactéria presente em efluentes domésticos) nos efluentes. No que denota o aspecto social, as populações que residem em torno das fábricas relataram que já contraíram doenças estomacais e dermatite alérgica em razão do contato direto com as águas residuais coloridas e que muitos dos seus animais também ficaram doentes após o contato. Ao final, enfatizaram a necessidade por novos estudos com foco nos impactos dessas manufatureiras à saúde humana e ao ambiente.

Já Yacout e Hassouna (2016) investigaram o destino (aterro sanitário ou incineração) fornecido aos resíduos sólidos da fábrica de fibra acrílica na região MENA (Oriente Médio e Norte da África), localizada em Alexandria no Egito. Os autores descreveram que ambas as escolhas resultariam em emissões atmosféricas $\left(\mathrm{CO}_{2}, \mathrm{NO}_{2}, \mathrm{CH} 4, \mathrm{NO}_{x}\right)$, contaminação do solo e da água (metais) com alta ecotoxicidade e potencial carcinogênico. Contudo, a deposição desses materiais é necessária e, entre as alternativas disponíveis, a incineração obteria melhor desempenho ambiental.

\subsection{Corantes}

Entre as substâncias existentes nos efluentes, os corantes são os principais contaminantes. Estima-se que $80 \%$ dos pigmentos são perdidos durante a produção (SANTOS et al., 2019). Os corantes são constituídos de grupos cromóforos ( $\mathrm{C}=\mathrm{O}$ cetona, $\mathrm{C}=\mathrm{N}$ carboamínico, $\mathrm{N}=\mathrm{N}$ azoíco, $\mathrm{N}=\mathrm{O}$ nitroso, $\mathrm{NO}_{2}=$ nitrito e $\mathrm{C}=\mathrm{C}$ etileno) que, ao absorverem os comprimentos de onda do espectro visível, fornecem a cor. Outro grupo 
importante são os auxocromos formados por grupos de hidroxila $(-\mathrm{OH})$, amino $\left(-\mathrm{NH}_{2}\right)$, aldeído $(-\mathrm{CHO})$ e metil mercaptano $\left(-\mathrm{CH}_{3}\right)$, que são responsáveis pela alteração do comprimento de onda absorvido pelos cromóforos, promovendo assim a intensidade da cor (MARTINS; SUCUPIRA; SUAREZ, 2015; SALLEH et al., 2011). Além disso, têm o papel de fixar o corante no objeto receptor dos pigmentos, determinando sua natureza, que pode ser básica ou ácida (ADEKOGE; BELLO, 2015).

Os corantes são classificados pelo modo de fixação à fibra (reativos, diretos, à cuba, enxofre, ácidos, azoicos, básicos, branqueadores, dispersivo, pré-metalizado), por sua estrutura química (antraquinona, trimetilmetano, xanteno, nitro, quinolina, flatocianinas) (BERRADI et al., 2019), ou através de sua carga em meio aquoso, sendo catiônicos aqueles com cargas positivas e aniônicos aqueles com cargas negativas.

Devido à composição desses efluentes, a Resolução CONAMA No 430/2011 regulamenta padrões, condições e diretrizes de lançamento das águas residuais no Brasil (BRASIL, 2011). Por isso, a necessidade das empresas em instalarem técnicas de tratamento de modo a atender às legislações vigentes e reduzir os impactos ao ambiente. Na literatura, encontram-se vários métodos, tais como processos físico-químicos (fotodegradação, ozônio, processo de oxidação avançada, processos eletroquímicos, separação de espuma, sonicação, adsorção, membrana, coagulação, floculação), processos biológicos (enzimas, leveduras, fungos, bactérias, algas) e processos combinados (físico-químicos e biológicos) (CHOUDRI; BAAWAIN, 2016). Dentre esses, o método de adsorção é considerado eficaz na remoção dos compostos orgânicos e inorgânicos existentes nos efluentes (SALLEH et al., 2011).

\subsection{Adsorção}

A adsorção é um processo de transferência de massa que utiliza a superfície de um sólido (adsorvente) para acumular substâncias (adsorvato) que estão presentes em fluido líquido ou gasoso. O tamanho da área superficial determina o desempenho da adsorção, pois quanto maior a área melhor a capacidade de adsorver o material (GISI et al., 2016; CORDA; KINI, 2018; BELLO et al., 2017). Assim, é fundamentada na alta porosidade e na superfície. De acordo com Vidal et al., diversos fatores podem interferir nesse processo, tais como: "[...] a área superficial, as propriedades do adsorvente e do adsorvato, a temperatura do sistema, a natureza do solvente e o pH do meio" (2014, p. 16). 
A interação entre adsorvato-adsorvente pode classificá-la em dois tipos: fisissorção ou quimissorção. A primeira, também denominada de adsorção física, ocorre quando o adsorvato se une à superfície do adsorvente com ligações fracas, o que pode ser atribuído às forças de Van der Waals. Já a adsorção química, ou quimissorção, é atribuída à troca ou compartilhamento de elétrons entre o adsorvato e a superfície do adsorvente, de modo que ocorra formação ou quebra de moléculas, permitindo nova ligação química. Desse modo, pode ser considerada bem mais forte que a fisissorção (ADEKOGE; BELLO, 2015; GISI et al., 2016). Outro aspecto considerável é que a fisissorção é não localizada, isto é, a substância pode aderir a qualquer local da área superficial, ao contrário da adsorção química, classificada como localizada, que permite que o adsorvato se ligue apenas aos sítios ativos presentes na área superficial (NASCIMENTO et al., 2014; ZHOU; ZHANG; CHENG, 2015).

Entre os adsorventes disponíveis, o carvão ativado (CA) é o mais usual. Esse material apresenta alto teor de carbono, porosidade e área superficial (CORDA; KINI, 2018). Várias matérias-primas podem ser transformadas em CA, tais como serragem de laranjeira (AZZAZ et al., 2017), casca de coco (AZAMAN et al., 2018), damasco (DJILANI et al., 2015), entre outras. Sua produção pode ser por ativação física ou química. Entre as aplicabilidades do CA, está o tratamento de efluentes e a purificação de água e gás. A depender do tamanho da partícula, pode ser classificado em: carvão ativado em pó (CA), carvão ativado granular (CAG) e carvão ativado por esferas (CAE) (CORDA; KINI, 2018).

Como mencionado anteriormente, a produção do CA pode ocorrer por ativação física ou química, a qual (simplificadamente) pode ser dividida em duas etapas: ativação e pirólise. A ativação física ocorre por meio da carbonização da matéria-prima, em atmosfera inerte, com temperatura entre 400 e $850^{\circ} \mathrm{C}$. Posteriormente, é submetido à ativação térmica, etapa em que se adicionam gases oxidantes, como $\mathrm{O}_{2}, \mathrm{CO}_{2}$ e vapor. Já a realização da ativação química sucede da aplicação de um reagente, como o ácido sulfúrico $\left(\mathrm{H}_{2} \mathrm{SO}_{4}\right)$, cloreto de zinco $\left(\mathrm{ZnCl}_{2}\right)$, ácido fosfórico $\left(\mathrm{H}_{3} \mathrm{PO}_{4}\right)$ e hidróxido de sódio $(\mathrm{NaOH})$, no agente precursor. Em sequência, ocorre o processo de pirólise com temperatura entre 400 e $850{ }^{\circ} \mathrm{C}$ (ADEKOGE; BELLO, 2015). Ao final, o CA é neutralizado a fim de remover as impurezas. Ressalta-se que a ativação química favorece a adsorção de moléculas menores, devido ao tamanho e volume dos poros. Em contrapartida, a ativação física adsorve melhor partículas maiores (GISI et al., 2016; NASCIMENTO et al., 2014). 


\subsection{Fontes carbonáceas investigadas para a produção de carvão ativado}

Através da busca bibliográfica, observou-se um número considerável de pesquisas direcionadas à aplicação direta de resíduos agrícolas para o tratamento de efluentes (CHOI; YU, 2019; DAHRI; KOOH; LIM, 2015; PAVLOVIĆ et al., 2015; RAHMAT et al., 2016; REDDY; NIRMALA; ASHWINI, 2017). Neste trabalho, o intuito da pesquisa foi a busca de fontes alternativas relatadas para a produção do carvão ativado. O resultado dessa busca é apresentado na Tabela 1. A escolha dos materiais derivados de resíduos agrícolas ocorreu devido a sua abundância, renovabilidade e baixo custo, podendo, assim, viabilizar a produção do adsorvente (BHATNAGAR; SILLANPÄÄ; WITEK-KROWIAK, 2015).

Na Tabela 1 são apresentadas as matérias-primas usadas na produção de carvão ativado entre os anos de 2014 a 2019. Nota-se a variação da área superficial, entre 1,86 a $1713 \mathrm{~m}^{2} \mathrm{~g}^{-}$ 1; e da capacidade de adsorção, entre 4,86 e 1365,10 $\mathrm{mg} \mathrm{g}^{-1}$. O azul de metileno foi a substância mais utilizada para os ensaios de adsorção, tal fato pode ser compreendido por ser considerado um corante modelo nos experimentos de remoção de poluentes em efluentes aquosos (RANGABHASHIYAM; ANU; SELVARAJU, 2013). Esses materiais são, portanto, alternativas atraentes para processos adsortivos.

Tabela 1: Matérias-primas empregadas na produção do carvão ativado para a remoção de uma variedade de corantes

\begin{tabular}{|c|c|c|c|c|c|}
\hline Matéria-prima & Ativação & $\begin{array}{c}\text { Área } \\
\text { superficial } \\
\left(\mathrm{m}^{2} \mathrm{~g}^{-1}\right)\end{array}$ & $\begin{array}{l}\text { Capacidade } \\
\text { adsorvativa } \\
\left(\mathrm{mg} \mathrm{g}^{-1}\right)\end{array}$ & Corante & Ref. \\
\hline $\begin{array}{c}\text { Irvingia } \\
\text { gabonenses }\end{array}$ & $\mathrm{H}_{2} \mathrm{SO}_{4}$ e $\mathrm{KOH}$ & 1,86 & 232,00 & Rodamina B & $\begin{array}{c}\text { (INYINBOR; } \\
\text { ADEKOLA; } \\
\text { OLATUNJI, 2015) }\end{array}$ \\
\hline Casca de avelã & $\mathrm{N}_{2}$ & 124,35 & 3,79 & \multirow{5}{*}{ Azul de Metileno } & $\begin{array}{c}\text { (KAYA; YILDIZ; } \\
\text { CEYLAN, 2018) }\end{array}$ \\
\hline $\begin{array}{c}\text { Guariroba } \\
\text { (Syagrus oleracea) }\end{array}$ & $\begin{array}{c}\mathrm{H}_{2} \mathrm{SO}_{4}, \mathrm{H}_{3} \mathrm{PO}_{4} \\
\text { e KOH }\end{array}$ & $\mathrm{N} / \mathrm{I}$ & 893,78 & & $\begin{array}{c}\text { (SANTOS et al., } \\
\text { 2019) }\end{array}$ \\
\hline $\begin{array}{c}\text { Cascas de } \\
\text { sementes de } \\
\text { Marula }\end{array}$ & $\mathrm{HCl}$ e $\mathrm{NaOH}$ & $\mathrm{N} / \mathrm{I}$ & 33,00 & & $\begin{array}{l}\text { (EDOKPAYI; } \\
\text { NDLOVU; } \\
\text { ODIYO,2019) }\end{array}$ \\
\hline $\begin{array}{c}\text { Castanha de Dão } \\
\text { (Ziziphus } \\
\text { mauritiana) }\end{array}$ & $\mathrm{KOH}$ e $\mathrm{HCl}$ & $\mathrm{N} / \mathrm{I}$ & 178,00 & & $\begin{array}{c}\text { (REGTI et al., } \\
\text { 2017) }\end{array}$ \\
\hline Bambu & Vapor e $\mathrm{N}_{2}$ & 734,00 & 53,40 & & $\begin{array}{l}\text { (HATA et al., } \\
\text { 2016) }\end{array}$ \\
\hline
\end{tabular}

Continua... 
... Continuação

\begin{tabular}{|c|c|c|c|c|c|}
\hline Matéria-prima & Ativação & $\begin{array}{c}\text { Área } \\
\text { superficial } \\
\left(\mathbf{m}^{2} \mathbf{g}^{-1}\right)\end{array}$ & $\begin{array}{l}\text { Capacidade } \\
\text { adsorvativa } \\
\left(\mathrm{mg} \mathrm{g}^{-1}\right)\end{array}$ & Corante & Ref. \\
\hline $\begin{array}{l}\text { Serragem de } \\
\text { Laranjeira }\end{array}$ & $\mathrm{NaOH}$ & $\mathrm{N} / \mathrm{I}^{*}$ & 78,74 & \multirow{3}{*}{ Azul de Metileno } & $\begin{array}{c}\text { (AZZAZ et al., } \\
\text { 2017) }\end{array}$ \\
\hline $\begin{array}{l}\text { Resíduos de } \\
\text { Pessegueiro }\end{array}$ & $\mathrm{H}_{3} \mathrm{PO}_{4}$ & 1521,00 & 600,20 & & $\begin{array}{c}\text { (ÁLVAREZ- } \\
\text { TORRELLAS et } \\
a l ., 2016)\end{array}$ \\
\hline $\begin{array}{c}\text { Casca de } \\
\text { Amendoim }\end{array}$ & $\mathrm{H}_{2} \mathrm{SO}_{4}$ & 3,70 & 1250,00 & & $\begin{array}{l}\text { (ISLAM et al., } \\
\text { 2019) }\end{array}$ \\
\hline Casca de coco & N/I & 1118,74 & 78,11 & \multirow[b]{2}{*}{ Verde Malaquita } & $\begin{array}{c}\text { (AZAMAN et al., } \\
\text { 2018) }\end{array}$ \\
\hline $\begin{array}{l}\text { Planta de } \\
\text { Beladona }\end{array}$ & $\begin{array}{c}\mathrm{ACS}: \mathrm{H}_{2} \mathrm{SO}_{4} \\
\\
\mathrm{AC} \mathrm{S} / \mathrm{N}: \\
\mathrm{H}_{2} \mathrm{SO}_{4} \text { e } \mathrm{HNO}_{3} \\
\\
\mathrm{AC}-\mathrm{S} / \mathrm{A}: \\
\left(\mathrm{NH}_{4}\right)_{2} \mathrm{~S}_{2} \mathrm{O}_{8} \mathrm{e} \\
\mathrm{H}_{2} \mathrm{SO}_{4}\end{array}$ & $\begin{array}{l}158,00 \\
266,00 \\
225,00\end{array}$ & $\begin{array}{l}100,00 \\
200,00 \\
125,00\end{array}$ & & $\begin{array}{c}\text { (RASHWAN et } \\
a l ., 2018)\end{array}$ \\
\hline Carvalho & $\mathrm{H}_{3} \mathrm{PO}_{4}$ e $\mathrm{HCl}$ & 1253,92 & $\begin{array}{l}40,16 \\
78,74\end{array}$ & $\begin{array}{l}\text { Vermelho congo } \\
\text { Alaranjado de } \\
\text { Metila }\end{array}$ & $\begin{array}{c}\text { (GHASEMIAN; } \\
\text { PALIZBAN, 2016) }\end{array}$ \\
\hline $\begin{array}{c}\text { Abacate (Persea } \\
\text { americana) }\end{array}$ & $\mathrm{KOH}$ & 1593,00 & 625,00 & Azul Básico 41 & $\begin{array}{c}\text { (REGTI et al., } \\
\text { 2017) }\end{array}$ \\
\hline Melia azedarach & $\mathrm{HCl}$ & $\mathrm{N} / \mathrm{I}$ & 12,92 & $\begin{array}{c}\text { Vermelho } \\
\text { Reativo } 141\end{array}$ & $\begin{array}{c}\text { (NAJAFPOOR } e t \\
a l ., 2016)\end{array}$ \\
\hline $\begin{array}{c}\text { Casca de abacate } \\
\text { (Persea } \\
\text { americana) }\end{array}$ & $\begin{array}{l}\mathrm{H}_{2} \mathrm{SO}_{4} \\
\mathrm{HNO}_{3}\end{array}$ & $\begin{array}{l}5,32 \\
8,45\end{array}$ & $\begin{array}{l}172,90 \\
182,70\end{array}$ & Vermelho procion & $\begin{array}{c}\text { (GEORGIN et } \\
a l ., 2018)\end{array}$ \\
\hline $\begin{array}{c}\text { Quinoa } \\
\text { (Chenopodium } \\
\text { quinoa Willd) }\end{array}$ & $\mathrm{KOH}$ e $\mathrm{HCl}$ & 1713,00 & $\begin{array}{l}759,39 \\
600,66 \\
561,72 \\
550,54 \\
1365,10\end{array}$ & $\begin{array}{c}\text { Rodamina B } \\
\text { Violeta de Metila } \\
\text { Azul de Metileno } \\
\text { Alaranjado de } \\
\text { Metila } \\
\text { Verde Malaquita }\end{array}$ & $\begin{array}{c}\text { (CHEN et al., } \\
\text { 2018) }\end{array}$ \\
\hline $\begin{array}{l}\text { Casca de romã } \\
\text { Casca de uva }\end{array}$ & $\mathrm{HCl}$ & $\mathrm{N} / \mathrm{I}$ & $\begin{array}{l}39,55 \\
45,20\end{array}$ & Azure A & $\begin{array}{l}\text { (GEÇIBESLER; } \\
\text { TOPRAK, 2017) }\end{array}$ \\
\hline
\end{tabular}

Continua... 
... Conclusão

\begin{tabular}{|c|c|c|c|c|c|}
\hline Matéria-prima & Ativação & $\begin{array}{c}\text { Área } \\
\text { superficial } \\
\left(\mathbf{m}^{2} \mathbf{g}^{-1}\right)\end{array}$ & $\begin{array}{c}\text { Capacidade } \\
\text { adsorvativa } \\
\left(\mathrm{mg} \mathrm{g}^{-1}\right)\end{array}$ & Corante & Ref. \\
\hline Casca de Toranja & $\mathrm{H}_{2} \mathrm{O}_{2}$ & N/I & 37,43 & $\begin{array}{l}\text { Sella Solid Blue } \\
\text { Special Violet } \\
\text { Derma Burdeaux } \\
\text { Sella Solid Orange }\end{array}$ & $\begin{array}{c}\text { (ROSALES et al., } \\
\text { 2016) }\end{array}$ \\
\hline $\begin{array}{l}\text { Resíduos do fruto } \\
\text { da Rapaneas } \\
\text { ferrugínea }\end{array}$ & $\begin{array}{c}\mathrm{C}_{2} \mathrm{H}_{5} \mathrm{OH} \\
\mathrm{H}_{2} \mathrm{SO}_{4}\end{array}$ & $\begin{array}{l}464,00 \\
368,00\end{array}$ & $\begin{array}{c}69,00 \text { e } 33,00 \\
106,00 \text { e } \\
125,00\end{array}$ & $\begin{array}{l}\text { Azul de Metileno } \\
\text { Violeta de Cristal }\end{array}$ & $\begin{array}{c}\text { (CHAHM; } \\
\text { MARTINS; } \\
\text { RODRIGUES, } \\
2018)\end{array}$ \\
\hline Nozes de damasco & $\mathrm{H}_{3} \mathrm{PO}_{4} \mathrm{e} \mathrm{HNO}_{3}$ & 359,40 & $\begin{array}{l}36,68 \\
32,25\end{array}$ & $\begin{array}{l}\text { Azul de Metileno } \\
\text { Alaranjado de } \\
\text { Metila }\end{array}$ & $\begin{array}{l}\text { (DJILANI et al., } \\
\text { 2015) }\end{array}$ \\
\hline $\begin{array}{c}\text { Sementes de } \\
\text { Groselha Indiana }\end{array}$ & & & 11,91 & & \\
\hline $\begin{array}{l}\text { Sementes de } \\
\text { Jamelão } \\
\text { Sementes de } \\
\text { Tamarindo }\end{array}$ & $\begin{array}{c}\mathrm{NaHCO}_{3} \mathrm{e} \\
\mathrm{HNO}_{3}\end{array}$ & N/I & 11,68 & Cromotrópicos & $\begin{array}{c}\text { (HAMEED; } \\
\text { MUTHIRULAN; } \\
\text { SUNDARAM, } \\
\text { 2017) }\end{array}$ \\
\hline $\begin{array}{c}\text { Sementes de } \\
\text { Acacia sinuata }\end{array}$ & & & 4,86 & & \\
\hline
\end{tabular}

*N/I = Não informado.

Fonte: Autoria própria.

Os materiais vegetais agrícolas são lignocelulósicos, constituídos de celulose, hemicelulose, lignina, extrativos, lipídios e hidrocarbonetos que contribuem para a sua massa molecular. A celulose, hemicelulose e lignina são os constituintes principais. A primeira, um polímero insolúvel em água e composto por anidroglucose, é importante para a formação da parede celular da biomassa. A segunda, uma fibra alimentar, é composta de diversos açúcares e encontrada em maior quantidade nas cascas e folhas. E por último, a lignina, que é um polímero do complexo aromático com estrutura tridimensional responsável pela sustentação da planta, condução da água das raízes às folhas e proteção contra a degradação da biomassa (BHATNAGAR; SILLANPÄÄ; WITEK-KROWIAK, 2015; MALIK; JAIN; YADAV, 2017).

Inyinbor, Adekola e Olatunji (2015) utilizaram a Irvingia gabonensis para a produção de CA por meio da ativação química, com ácido sulfúrico e hidróxido de potássio para 
remoção do corante rodamina B. O material obteve capacidade máxima adsortiva de 232,00 $\mathrm{mg} \mathrm{g}^{-1}$.

Para a remoção do corante azul de metileno ( $\mathrm{AM}$ ), várias pesquisas mostraram a transformação de diversos resíduos agrícolas em CA. Kaya, Yildiz e Ceylan (2018) utilizaram a casca de avelã por meio da ativação física. $\mathrm{O}$ material apresentou capacidade máxima adsortiva de $3,79 \mathrm{mg} \mathrm{g}^{-1}$. Santos et al. (2019) usaram o endocarpo da Guariroba (Syagrus oleracea) por meio da ativação química $\left(\mathrm{H}_{2} \mathrm{SO}_{4}, \mathrm{H}_{3} \mathrm{PO}_{4}\right.$ e $\left.\mathrm{KOH}\right)$ e constataram capacidade máxima adsortiva de $893,78 \mathrm{mg} \mathrm{g}^{-1}$. Além disso, consideraram o material eficiente na remoção de efluentes coloridos.

Edokpayi, Ndlovu e Odiyo (2019) preparam o CA oriundo das cascas das sementes de Marula com ativação química $(\mathrm{HCl}$ e $\mathrm{NaOH})$ para adsorção do $\mathrm{AM}$. O estudo apresentou capacidade máxima adsortiva de $33 \mathrm{mg} \mathrm{g}^{-1}$. Regti et al. (2017) verificaram a capacidade das nozes de Ziziphus mauritiana com tratamento químico na remoção de AM e constataram a relevância dos adsorventes no tratamento de corantes, apresentando capacidade máxima adsortiva de $178 \mathrm{mg} \mathrm{g}^{-1}$. Hata et al. (2016) trataram por ativação térmica o bambu e informaram que o material utilizado apresentou capacidade máxima adsortiva de 53,40 mg $\mathrm{g}^{-1}$.

Azzaz et al. (2017) estudaram a serragem de laranjeira ativada com hidróxido de sódio e notaram que o CA produzido obteve capacidade máxima adsortiva de 78,74 $\mathrm{mg} \mathrm{g}^{-1}$. Álvarez-Torrellas et al. (2016) analisaram a capacidade de remoção do AM através do CA derivado de resíduos de pessegueiro com ativação química $\left(\mathrm{H}_{3} \mathrm{PO}_{4}\right)$, obtendo capacidade máxima adsortiva de $600,20 \mathrm{mg} \mathrm{g}^{-1}$. Islam et al. (2019) preparam o CA derivado da casca de amendoim ativado com ácido sulfúrico. Os resultados demonstraram capacidade máxima adsortiva de $1250 \mathrm{mg} \mathrm{g}^{-1}$.

Azaman et al. (2018) adquiriram carvão ativado derivado do coco para o tratamento do corante verde malaquita (VM). O CA exibiu capacidade máxima adsortiva de 78,11 mg $\mathrm{g}^{-1}$. Rashwan et al. (2018) forneceram tratamentos químicos diferentes para a transformação das folhas de Atropa belladonna para remoção do VM. O material apresentou capacidade máxima adsortiva de até $200 \mathrm{mg} \mathrm{g}^{-1}$.

Para a adsorção do vermelho congo (VC) e alaranjado de metila (AM), Ghasemian e Palizban (2016) empregaram o CA oriundo de carvalho, impregnado com nanopartículas de carboneto de silício. O estudo obteve capacidade máxima adsorvativa de 40,16 $\mathrm{mg} \mathrm{g}^{-1}$ e 78,4 $\mathrm{mg} \mathrm{g}^{-1}$ para o VC e AM respectivamente. Regti et al. (2017) empregaram as cascas de 
abacate (Persea americana) com tratamento químico $\left(\mathrm{HNO}_{3}\right.$ e $\left.\mathrm{KOH}\right)$ para remoção do azul básico 41 . O estudo apresentou capacidade máxima de adsorção de $625 \mathrm{mg} \mathrm{g}^{-1}$.

Najafpoor et al. (2016) realizaram tratamento químico $(\mathrm{HCl})$ da serragem de Melia Azaderach para obtenção do CA a fim de remover o corante vermelho reativo 141. A capacidade máxima de adsorção foi de $12,92 \mathrm{mg} \mathrm{g}^{-1}$.

Georgin et al. (2018) utilizaram as cascas de abacate com ativação química $\left(\mathrm{H}_{2} \mathrm{SO}_{4}\right.$ e $\mathrm{HNO}_{3}$ ) para a produção de CA a fim de remover o vermelho procion. Os autores relataram que o CA apresentou capacidade máxima de adsorção de $172,90 \mathrm{mg} \mathrm{g}^{-1}$ e $182,70 \mathrm{mg} \mathrm{g}^{-1}$, tendo, assim, um bom desempenho na remoção de compostos orgânicos.

Chen et al. (2018) preparam a casca de quinoa com ativação química (KOH e $\mathrm{HCl})$ para adsorver os corantes rodamina $\mathrm{B}$, violeta de metila, azul de metileno, alaranjado de metila e verde malaquita. Após as análises, o material obteve capacidade máxima de adsorção de 759,39, 600,66, 561,72, 550,54 e 1365,10 $\mathrm{mg} \mathrm{g}^{-1}$, respectivamente. Geçibesler e Toprak (2017) modificaram quimicamente ( $\mathrm{HCl})$ as hastes de uva e casca de romã para remoção do Azure A. No estudo, observaram que o adsorvente obteve capacidade máxima adsortiva de 45,20 $\mathrm{mg} \mathrm{g}^{-1}$ e 39,55 $\mathrm{mg} \mathrm{g}^{-1}$, respectivamente. Além disso, consideraram que os materiais ecofriendlys são eficientes na remoção do corante.

Rosales et al. (2016) usaram as cascas de toranja tratadas com peróxido de hidrogênio para remoção do sella solid blue, special violet, derma burdeaux e sella solid orange. Assim, concluíram que a capacidade máxima adsortiva foi de $37,43 \mathrm{mg} \mathrm{g}^{-1}$. Chahm, Martins, Rodrigues (2018) demonstraram a eficiência dos resíduos da fruta de Rapanea ferruginea tratados quimicamente $\left(\mathrm{C}_{2} \mathrm{H}_{5} \mathrm{OH}\right.$ e $\left.\mathrm{H}_{2} \mathrm{SO}_{4}\right)$ para a remoção de azul de metileno e violeta de cristal. O adsorvente apresentou capacidade máxima de adsorção de 33,00, 69,00, 106,00 e $125,00 \mathrm{mg} \mathrm{g}^{-1}$, respectivamente.

Djilani et al. (2015) estudaram as propriedades do CA oriundo das nozes de damasco com ativação química para remoção do azul de metileno e alaranjado de metila. Os autores consideraram que as nozes de damasco obtiveram 36,68 $\mathrm{mg} \mathrm{g}^{-1}$ e 32,25 $\mathrm{mg} \mathrm{g}^{-1}$ de capacidade máxima de adsorção.

Hameed, Muthirulan e Sundaram (2017) investigaram a capacidade das sementes de groselha indiana, jamelão, tamarindo e Acacia sinuata tratadas com $\mathrm{NaHCO}_{3}$ e $\mathrm{HNO}_{3}$ para obtenção do CA. Os adsorventes apresentaram capacidade máxima adsortiva de 11,91; 11,$68 ; 5,00$ e $4,86 \mathrm{mg} \mathrm{g}^{-1}$, respectivamente. 


\section{CONSIDERAÇÕES FINAIS}

Esta revisão enfatizou a necessidade do tratamento de efluentes e demonstrou diversos resíduos agrícolas investigados para produção do carvão ativado. Esses materiais lignocelulósicos são abundantes, renováveis e de baixo custo, sendo definidos como ecofriendly. O potencial na remoção de diferentes corantes têxteis foi relatado em diversas pesquisas.

\section{REFERÊNCIAS}

ADEKOGE, K. A.; BELLO, O. S. Dye sequestration using agricultural wastes as adsorbents. Water Resources and Industry. v. 12, p. 8-24, dez. 2015. Disponível em: https://doi.org/10.1016/j.wri.2015.09.002 . Acessado em: 15 jun. 2019.

ÁLVAREZ-TORRELLAS, S.; OVEJERO, G.; GARCÍA-LOVERA, R.; RODRÍGUEZ, A.; GARCÍA, J. Synthesis of a mesoporous carbon from peach stones for adsorption of basic dyes from wastewater: kinetics, modeling, and thermodynamic studies. Clean Technologies and Environmental Policy. v. 18, p. 1085-1096, 2016. Disponível em: https://doi.org/10.1007/s10098-016-1098-y. Acessado em: 16 jun. 2019.

AZZAZ, A. A.; JELlALI, S.; AKROUT, H.; ASSADI, A. A.; BOUSSELMI, L. Optimization of a cationic dye removal by a chemically modified agriculture byproduct using response surface methodology: biomasses characterization and adsorption properties. Environmental Science and Pollution Research. v. 24, p. 9831-9846, 2017. Disponível em: https://doi.org/10.1007/s11356-016-7698-6. Acessado em: 15 jun. 2019.

AZAMAN, S. A.H.; AFANDI, A.; HAMEED, B. H.; MOHD DIN, A. T. Removal of Malachite Green from Aqueous Phase Using Coconut Shell Activated Carbon: Adsorption, Desorption, and Reusability Studies. Journal of Applied Science and Engineering. v. 21, n. 3, p. 317-330, 2018. Disponível em: http://jase.tku.edu.tw/21-3/03C10604_0281.pdf. Acessado em: 15 jun. 2019.

BELLO, I. A.; KABBASHI, N. A.; ALAM, MD. Z.; ALKHATIB, M'AN. F.; 1 MURAD, F. N,; QUDSIEH, I. Y. Challenges in textile wastewater and current palliative methods: an overview. IIUM Engineering Journal. v. 18, n. 2, p. 71-78, 2017. Disponível em: https://doi.org/10.31436/iiumej.v18i2.742. Acessado em: 15 jun. 2019.

BERRADI, M.; HSISSOU, R.; KHUDHAIR, M.; ASSOUANG, M.; CHERKAOUI, O.; BACHIRI, A. E.; HARFI, A. E. Textile finishing dyes and their impact on aquatic environs. Heliyon, v. 5, p. e02711, 2019. Disponível em: https://dx.doi.org/10.1016\%2Fj.heliyon.2019.e02711. Acessado em: 18 abr. 2020. 
BHATNAGAR, A.; SILLANPÄÄ, M.; WITEK-KROWIAK, A. Agricultural waste peels as versatile biomass for water purification - A review. Chemical Engineering Journal. v. 270, p. 244-271, jun. 2015. Disponível em: https://doi.org/10.1016/j.cej.2015.01.135. Acessado em: 17 jun. 2019.

BRASIL. CONAMA No 430/2011. Dispõe sobre condições e padrões de lançamento de efluentes, complementa e altera a Resolução no 357, de 17 de março de 2005, do Conselho Nacional do Meio Ambiente - CONAMA. Disponível em: http://www2.mma.gov.br/port/conama/legiabre.cfm?codlegi=646. Acessado em: 13 jul. 2019.

BRILLAS, E.; MARTÍNEZ-HUITLE, C. A. Decontamination of wastewaters containing synthetic organic dyes by electrochemical methods. An updated review. Applied Catalysis B: Environmental. v. 166-167, p. 603-643, maio. 2015. Disponível em: https://doi.org/10.1016/j.apcatb.2014.11.016. Acessado em: 15 jun. 2019.

CAPES. Periódicos da Coordenação de Aperfeiçoamento de Pessoal de Nível Superior Disponível em: https://www.periodicos.capes.gov.br/?option=com_pcollection\&mn=70\&smn=79\&cid=81. Acessado em: 31 maio 2019.

CORDA, N. C.; KINI, M. S. A Review on Adsorption of Cationic Dyes using Activated Carbon. MATEC Web of Conferences. v. 144, p. 02022, jan. 2018. Disponível em: https://doi.org/10.1051/matecconf/201814402022. Acessado em: 10 jul, 2019.

CHAHM, T.; MARTINS, B. A.; RODRIGUES, C. A. Adsorption of methylene blue and crystal violet on low-cost adsorbent: waste fruits of Rapanea ferruginea (ethanoltreated and $\mathrm{H}_{2} \mathrm{SO}_{4}$-treated). Environmental Earth Sciences. v. 77, p. 508, jun. 2018. Disponível em: https://doi.org/10.1007/s12665-018-7681-2. Acessado em: 27 jul. 2019.

CHEN, S.; TANG, S.; SUN, Y.; WANG, G.; CHEN, H.; YU, X.; SU, Y.; CHEN, G. Preparation of a Highly Porous Carbon Material Based on Quinoa Husk and Its Application for Removal of Dyes by Adsorption. Materials. v. 11, n. 8, p. 1407. jun. 2018. Disponível em: https://doi.org/10.3390/ma11081407. Acessado em: 11 jun, 2019.

CHICATTO, J. A; NUNES, H. C. A.; GONÇALVES, M. J.; HELM, C. V.; VAZ, D. A.; TAVAREZ, L. B. B. Strategies for decolorization of textile industry effluents by whiterot-fungi with peach palm residue. Acta Scientiarum. Technology. v. 40, n. 1, p. e35610, abr. 2018. Disponível em: https://doi.org/10.4025/actascitechnol.v40i1.35610. Acessado em: 03 jul. 2019.

CHOI, H-J.; YU, S-W. Biosorption of methylene blue from aqueous solution by agricultural bioadsorbent corncob. Environmental Engineering Research. v. 24, n. 1, p. 99-106, jun. 2019. Disponível em: http://eeer.org/journal/view.php?doi=10.4491/eer.2018.107. Acessado em: 11 jul. 2019.

CHOUDRI, B. S.; BAAWAIN, M. Textiles. Water Environment Research. v. 87, n. 10, p. 1433-1445, out. 2016.2 Disponível em: https://doi.org/10.2175/106143016X14696400495172. Acessado em: 15 jul. 2019. 
CROCE, R.; CINÀ, F.; LOMBARDO, A.; CRISPEYN, G.; CAPPELLI, C. I.; VIAN, M.; MAIORANA, S.; BENFENATI1, E.; BADERNA, D. Aquatic toxicity of several textile dye formulations: Acute and chronic assays with Daphnia magna and Raphidocelis subcapitata. Ecotoxicology and Environmental Safety, v. 144, p. 79-87, 2017. Disponível em: https://doi.org/10.1016/j.ecoenv.2017.05.046. Acessado em: 18 abr. 2020.

DADI, D.; STELMACHER, T.; SENBETA, F,; PASSEL, S. V.; AZZADI, H. Environmental and health impacts of effluents from textile industries in Ethiopia: the case of Gelan and Dukem, Oromia Regional State. Environmental Monitoring and Assessment. v.189, p.11, 2017. Disponível em: https://doi.org/10.1007/s10661-016-5694-4. Acessado em: 15 jul. 2019.

DAHRI, M. K.; KOOH, M. R. R.; LIM, L. B. L. Application of Casuarina equisetifolia needle for the removal of methylene blue and malachite green dyes from aqueous solution. Alexandria Engineering Journal. v. 54, n. 4, p. 1253-1263, dez. 2015. Disponível em: https://doi.org/10.1016/j.aej.2015.07.005. Acessado em: 13 jul. 2019.

DASGUPTA, J.; SIKDER, J.; CHAKRABORTY, S.; CURCIO, S.; DRIOLI, E. Remediation of textile effluents by membrane based treatment techniques: A state of the art review. Journal of Environmental Management. v. 147, p. 55-72, jan. 2015. Disponível em: https://doi.org/10.1016/j.jenvman.2014.08.008. Acessado em: 16 jul. 2019.

DJILANI, C.; ZAGHDOUDI, R.; DJAZI, F.; BOUCHEKIMA, B.; LALLAM, A.; MODARRESSI, A.; ROGALSKI, M. Adsorption of dyes on activated carbon prepared from apricot stones and commercial activated carbon. Journal of the Taiwan Institute of Chemical. v. 53, p. 112-121, ag. 2015. Disponível em: https://doi.org/10.1016/j.jtice.2015.02.025. Acessado em: 16 jul. 2019.

EDOKPAYI, J. N.; NDLOVU, S. S.; ODIYO, J. O. Characterization of pulverized Marula seed husk and its potential for the sequestration of methylene blue from aqueous solution. BMC Chemistry. v. 13, n. 10, jan. 2019. Disponível em: https://doi.org/10.1186/s13065-019-0530-x. Acessado em: 17 jul. 2019.

GAUTAM, R. K.; GAUTAM, P. K.; BARNEJEE, S.; RAWAT, V.; SONI, S.; SHARMA, S. K.; CHATTOPADHYAYA. Removal of tartrazine by activated carbon biosorbents of Lantana camara: Kinetics, equilibrium modeling and spectroscopic analysis. Journal of Environmental Chemical Engineering. v. 3, p. 79-88, mar. 2015. Disponível em: https://doi.org/10.1016/j.jece.2014.11.026. Acessado em: 16 jul. 2019.

GEÇIBESLER, İ. H.; TOPRAK, M. Azure A Removal from Aqueous System Using Natural and Modified (Grape Stalk and Pomegranate Peel) Adsorbents. Proceedings of the National Academy of Sciences, India Section A: Physical Sciences. v. 87, p. 171-179, jun. 2017. Disponível em: https://doi.org/10.1007/s40010-017-0352-y. Acessado em: 22 jul. 2019.

GEORGIN, J.; MARQUES, B. S.; SALLA, J. S.; FOLETTO, E. L.; ALLASIA, D.; DOTTO, G. L. Removal of Procion Red dye from colored effluents using $\mathrm{H}_{2} \mathrm{SO}_{4} / \mathrm{HNO}_{3}$ treated avocado shells (Persea americana) as adsorbent. Environmental Science and Pollution Research. v. 25, p. 6429-6442, mar. 2018. Disponível em: https://doi.org/10.1007/s11356017-0975-1. Acessado em: 16 jul. 2019. 
GISI, D. S.; LOFRANO, G., GRASSI, M.; NOTARMICOLA, M. Characteristics and adsorption capacities of low-cost sorbents for wastewater treatment: A review. Sustainable Materials and Technologies. v. 9, p. 10-40, set. 2016. Disponível em: https://doi.org/10.1016/j.susmat.2016.06.002. Acessado em: 12 jul. 2019

GHASEMIAN, E.; PALIZBAN, Z. Comparisons of azo dye adsorptions onto activated carbon and silicon carbide nanoparticles loaded on activated carbon. International Journal of Environmental Science and Technology. v. 13, p. 501-512, fev. 2016. Disponível em: https://doi.org/10.1007/s13762-015-0875-1. Acessado em: 15 jul. 2019.

HAMEED, K. S.; MUTHIRULAN, P.; SUNDARAM, M. M. Adsorption of chromotrope dye onto activated carbons obtained from the seeds of various plants: Equilibrium and kinetics studies. Arabian Journal of Chemistry. v. 10, n. 2, p. S2225-S2233, maio. 2017. Disponível em: https://doi.org/10.1016/j.arabjc.2013.07.058. Acessado em: 21 jul. 2019.

HATA, M.; AMANO, Y.; THIRAVETYAN, P.; MACHIDA, M. Preparation of Bamboo Chars and Bamboo Activated Carbons to Remove Color and COD from Ink Wastewater. Water Environment Research, v. 88, p. 87-96, 2016. Disponível em: https://doi.org/10.2175/106143015X14362865227751. Acessado em: 16 jul. 2019.

INYINBOR, A. A; ADEKOLA, F. A.; OLATUNJI,G. A. Adsorption of Rhodamine B Dye from Aqueous Solution on Irvingia gabonensis Biomass: Kinetics and Thermodynamics Studies. South African Chemical Institute. v. 68, p. 115-125, 2015. Disponível em: http://dx.doi.org/10.17159/0379-4350/2015/V68A17. Acessado em: 27 jul. 2019.

ISLAM, M. T.; HYDER, A. G.; SAENZ-ARANA, R.; HERNANDEZ, C.; GUINTO, T.; AHSAN, M. A.; ALVARADO-TENORIO, B.; NOVERON, J. C. Removal of methylene blue and tetracycline from water using peanut shell derived adsorbent prepared by sulfuric acid reflux. Journal of Environmental Chemical Engineering. v. 7, p. 102816, fev. 2019. Disponível em: https://doi.org/10.1016/j.jece.2018.102816. Acessado em: 22 jul. 2019.

KAYA, N.; YILDIZ, Z.; CEYLAN, S. Preparation and characterisation of biochar from hazelnut shell and its adsorption properties for methylene blue dye. Politeknik Dergisi. v. 21, p. 765-776, 2018. Disponível em: https://dergipark.org.tr/tr/download/articlefile/415114. Acessado em: 27 jul. 2019.

MALIK, D. S.; JAIN, C. K.; YADAV, A. K. Removal of heavy metals from emerging cellulosic low-cost adsorbents: a review. Applied Water Science. v. 7, p. 2113-2136, set. 2017. Disponível em: https://doi.org/10.1007/s13201-016-0401-8. Acessado em: 22 jul. 2019.

MARTINS, G. B. C.; SUCUPIRA, R. R.; SUAREZ, P. A. Z. A Química e as Cores. Revista Virtual Quimica. v. 7, p. 1508-1534, maio. 2015. Disponívele em: http://dx.doi.org/10.5935/1984-6835.20150082. Acessado em: 17 jul. 2019.

NAJAFPOOR, A.; ALIDADI, H.; ESMAEILI, H.; HADILOU, T.; DOLATABADI, M.; HOSSEINZADEH, A.; DAVOUDI, M. Optimization of anionic dye adsorption onto Melia azedarach sawdust in aqueous solutions: effect of calcium cations. Asia-Pacific Journal of Chemical Engineering. v. 11, p. 258-270, dez. 2016. Disponível em: https://doi.org/10.1002/apj.1962. Acessado em: 18 jul. 2019. 
NASCIMENTO, R. F. et al. Adsorção: aspectos teóricos e aplicações ambientais. Fortaleza: Imprensa Universitária, p. 256, 2014.

PAVLOVIĆ, M. D.; NIKOLIĆ, I. R.; MILUTINOVIĆ, M. D.; DIMITRIJEVIĆBRANKOVIĆ, S. I.; ŠILER-MARINKOVIĆ, S. S; ANTONOVIĆ, D. G. Plant waste materials from restaurants as the adsorbents for dyes. Hemijska industrija. v. 69, p. 667677, 2015. Disponível em: http://www.doiserbia.nb.rs/Article.aspx?ID=0367598X1400089P\#.XphTxchKhPY. Acessado em: 27 jul. 2019.

RAHMAT, N. A.; ALI, A. A.; SALMIATI; HUSSAIN, N.; MUHAMAD, M. S.; KRISTANTI, R. A.; HADIBARATA, T. Removal of Remazol Brilliant Blue $\mathbf{R}$ from Aqueous Solution by Adsorption Using Pineapple Leaf Powder and Lime Peel Powder. Water, Air \& Soil Pollution, v. 227, p. 105, 2016. Disponível em https://doi.org/10.1007/s11270-016-2807-1. Acessado em: 19 jul. 2019.

RANGABHASHIYAM, S.; ANU, N; SELVARAJU, N. Sequestration of dye from textile industry wastewater using agricultural waste products as adsorbents. Journal of Environmental Chemical Engineering, v. 1, p. 629-641, 2013. Disponível em: https://doi.org/10.1016/j.jece.2013.07.014. Acessado em: 27 jul. 2019.

RASHWAN, W. E.; EL-NABARAWY, T.; ABOELENEIN, R. M.; FATHY, N. A. Effect of Chemical Oxidation on the Adsorption Properties of Cationic Dye on Activated Carbons Prepared from Locally Atropa belladonna. Egyptian Journal of Chemistry, v. 61, p. 517-530, 2018. Disponível em: https://dx.doi.org/10.21608/ejchem.2018.2954.1259. Acessado em: 22 de jul. 2019.

REDDY, M. C. S.; NIRMALA, V.; ASHWINI, C. Bengal Gram Seed Husk as an adsorbent for the removal of dye from aqueous solutions - Batch studies. Arabian Journal of Chemistry, v. 10, p. S2554-S2566, 2017. Disponível em: https://doi.org/10.1016/j.arabjc.2013.09.029. Acessado em: 25 jul. 2019.

REGTI, A.; LAAMARI, M. R.; STIRIBA, S-E.; EL HADDAD, M. The potential use of activated carbon prepared from Ziziphus species for removing dyes from waste waters. Applied Water Science, v. 7, p. 4099-4108, 2017. Disponível em: https://doi.org/10.1007/s13201-017-0567-8. Acessado em: 25 jul. 2019.

REGTI, A.; LAAMARI, M. R.; STIRIBA, S-E.; EL HADDAD, M. Removal of Basic Blue 41 dyes using Persea americana-activated carbon prepared by phosphoric acid action. International Journal of Industrial Chemistry, v. 8, p. 187-195, 2017. Disponível em: https://doi.org/10.1007/s40090-016-0090-z. Acessado em: 22 jul. 2019.

ROSALES, E.; MEIJIDE, J.; TAVARES, T.; PAZOS, M.; SANROMÁN, M.A. Grapefruit peelings as a promising biosorbent for the removal of leather dyes and hexavalent chromium. Process Safety and Environmental Protection, v. 101, p. 61-71, 2016. Disponível em: https://doi.org/10.1016/j.psep.2016.03.006. Acessado em: 22 jul. 2019.

SALLEH, M. A. M.; MAHMOUD, D. K.; KARIM, W. A. W. A.; IDRIS, A. Cationic and anionic dye adsorption by agricultural solid wastes: A comprehensive review. Desalination, v. 280, p. 1-13, 2011. Disponível em: https://doi.org/10.1016/j.desal.2011.07.019. Acessado em: 21 jul. 2019. 
SANTOS, R. F.; RAMLOW, H.; DOLZAN, N.; MACHADO, R. A. F.; AGUIAR, C. R. L.; MARANGONI, C. Influence of different textile fibers on characterization of dyeing wastewater and final effluent. Environmental Monitoring and Assessment, v. 190, p. 693, 2019. Disponível em: https://doi.org/10.1007/s10661-018-7068-6. Acessado em: 24 jul. 2019.

SANTOS, K. J. L.; SANTOS, G. E. S.; SÁ, Í. M. G. L.; CARVALHO, S. H. V.; SOLETTI, J. I.; MEILI, L.; DUARTE, J. L. S.; BISPO, M. D.; DOTTO, G. L. Syagrus oleraceaactivated carbon prepared by vacuum pyrolysis for methylene blue adsorption. Environmental Science and Pollution Research, v. 26, p. 16470-16481, 2019. Disponível em: https://doi.org/10.1007/s11356-019-05083-4. Acessado em: 22 jul. 2019.

SHANKER, U.; RANI, M.; JASSAL, V. Degradation of hazardous organic dyes in water by nanomaterials. Environmental Chemistry Letters, v. 15, p. 623-642, 2017. Disponível em: https://doi.org/10.1007/s10311-017-0650-2. Acessado em: 23 jul. 2019.

SMITHA, T.; SANTHI, T.; PRASAD, A. L.; MANONMANI, S. Cucumis sativus used as adsorbent for the removal of dyes from aqueous solution. Arabian Journal of Chemistry, v. 10, p. S244-S251, 2017. Disponível em: https://doi.org/10.1016/j.arabjc.2012.07.030. Acessado em: 23 jul. 2019.

SUBRAMANIAM, R.; PONNUSAMY, K. S. Novel adsorbent from agricultural waste (cashew NUT shell) for methylene blue dye removal: Optimization by response surface methodology. Water Resources and Industry, v. 11, p. 64-70, 2015. Disponível em: https://doi.org/10.1016/j.arabjc.2012.07.030. Acessado em: 24 jul. 2019.

VIDAL CB, LIMA ACA, MELO DQ, RAULINO GSC, NASCIMENTO RF. Cinética de Adsorção. In: Nascimento RF et al. (org) Adsorção: aspectos teóricos e aplicações ambientais. Fortaleza: Imprensa Universitária, 2014, p. 256. Disponível em: http://www.repositorio.ufc.br/handle/riufc/10267. Acessado em: 21 abr. 2020.

SIVARAJASEKAR, N.; BASKAR, R.; RAGU, T.; SARIKA, K.; PREETHI, N.; RADHIKA, T. Biosorption studies on waste cotton seed for cationic dyes sequestration: equilibrium and thermodynamics. Applied Water Science, v. 7, p. 1987-1995, 2017. Disponível em: https://doi.org/10.1007/s13201-016-0379-2. Acessado em: 27 jul. 2019.

YACOUT, D. M. M.; HASSOUNA, M. S. Identifying potential environmental impacts of waste handling strategies in textile industry. Environmental Monitoring and Assessment, v. 188, p. 445, 2016. Dipsonível em: https://doi.org/10.1007/s10661-016-54438. Acessado em: 23 jul. 2019.

YASEEN, D. A.; SCHOLZ, M. Textile dye wastewater characteristics and constituents of synthetic effluents: a critical review. International Journal of Environmental Science and Technology, v. 16, p. 1193-1226, 2019. Disponível em: https://doi.org/10.1007/s13762018-2130-z. Acessado em: 23 jul. 2019.

WANASSI, B.; HARIZ, I. B.; GHIMBEU, C. M.; VAULOT, C.; HASSEM, M. B.; JEGUIRIM, M. Carbonaceous adsorbents derived from textile cotton waste for the removal of Alizarin $S$ dye from aqueous effluent: kinetic and equilibrium studies. 
Environmental Science and Pollution Research, v. 24, p. 10041-10055, 2017. Disponível em: https://doi.org/10.1007/s11356-017-8410-1. Acessado em: 24 jul. 2019.

ZHOU, Y.; ZHANG, L.; CHENG, Z. Removal of organic pollutants from aqueous solution using agricultural wastes: a review. Journal of Molecular Liquids, v. 212, p. 739762, 2015. Disponível em: https://doi.org/10.1016/j.molliq.2015.10.023. Acessado em: 25 jul. 2019. 